\title{
Mucosa mordiscada: relato de caso
}

Nibbled mucosa: case report

Mordedura de mucosa: reporte de caso

\begin{abstract}
Wanderley Barros dos Santos ${ }^{1 *}$, Carlos Sousa Mello de Almeida1, Carlos Eduardo Campos Ramos ${ }^{1}$, Lucas Emanuel Bezerra Araújo Fernandes', Weizia Gomes da Rocha1, Renata da Silva Pereira ${ }^{1}$, Katharina Jucá de Moraes Fernandes'1.
\end{abstract}

\section{RESUMO}

Objetivo: Relatar o caso de clínico de mucosa mordiscada associada a hábitos parafuncionais e possível transtorno de ansiedade, apresentando suas características clínicas, diagnóstico diferencial e manejo proposto. Detalhamento do caso: Paciente do gênero masculino, 15 anos, buscou atendimento após ser encaminhado por seu médico, com lesões nas regiões de mucosa jugalbilateral, lábio inferior e borda lateral direita de língua. Após anamnese e exame físico, foi observado que o paciente não possuía sinais e sintomas da disfunção temporomandibular, apresentando apenas as lesões nas regiões citadas, causada pelo hábito parafuncional. A terapia indicada para o paciente foi a orientação de cessar os hábitos parafuncionais para regressão da lesão, prescrição de pomada para aplicação tópica e encaminhamento para a clínica de psicologia. Considerações finais: Torna-se importante o correto diagnóstico dessas lesões orais, tal como a correlação com a história médica através de uma anamnese precisa, a fim de orientar a correção de hábitos parafuncionais e acompanhamento multidisciplinar quando necessário.

Palavras-chave: Mucosa bucal, Ansiedade, Depressão, Saúde bucal.

\section{ABSTRACT}

Objective: To report the case of nibbled mucosa associated with parafunctional habits and possible anxiety disorder, presenting its clinical characteristics, differential diagnosis and clinical management. Case Detail: A 15-year-old male patient sought care after being referred by the doctor, with lesions in bilateral cheek mucosa, lower lip and right lateral border of the tongue regions. After anamnesis and physical examination, it was observed that the patient had no signs and symptoms of temporomandibular dysfunction, presenting only the lesions in the mentioned regions, caused by parafunctional habit. The therapy indicated for the patient was the orientation to interrupt the parafunctional habits of regression of the lesion, the prescription of ointment for topical application and the referral to the psychology clinic. Final considerations: It is important to correctly diagnose these oral lesions as well as the adjustment with an accurate medical history, to guide the correction of parafunctional habits and multidisciplinary monitoring when necessary.

Keywords: Mouth mucosa, Anxiety, Depression, Oral health.

\section{RESUMEN}

Objetivo:Informe el caso de un médico con mordedura de mucosa asociada con hábitos parafuncionales y posible trastorno de ansiedad, presentando sus características clínicas, diagnóstico diferencial y manejo propuesto.Detalle del caso: Un paciente masculino de 15 años buscó atención después de ser referido por

${ }^{1}$ Centro Universitário (CESMAC), Maceió-Alagoas. *E-mail: wanderley.barros108@gmail.com 
su médico, con lesiones en la mucosa yugal bilateral, labio inferior y borde lateral derecho de las regiones de la lengua. Después de la anamnesis y el examen físico, se observó que el paciente no tenía signos y síntomas de disfunción temporomandibular, presentando solo las lesiones en las regiones mencionadas, causadas por hábito parafuncional. La terapia indicada para el paciente fue la orientación para detener los hábitos parafuncionales para la regresión de la lesión, la prescripción de ungüento para aplicación tópica y la derivación a la clínica de psicología. Consideraciones finales: Es importante diagnosticar correctamente estas lesiones orales, así como la correlación con el historial médico a través de un historial preciso, para guiar la corrección de los hábitos parafuncionales y el seguimiento multidisciplinario cuando sea necessário.

Palabras clave: Mucosa bucal, Ansiedad, Depresión, Salud bucal.

\section{INTRODUÇÃO}

Morsicatio buccarum é o termo científico empregado para mastigação crônica da bochecha, que normalmente leva a exposição tecidual com áreas descamativas, resultando em áreas com fragmentos de queratina, dando o aspecto esbranquiçado na superfície da mucosa. Essas lesões comumente são localizadas na mucosa jugal, entretanto, locais como mucosa labial (morsicatio labiorum) e margem lateral de língua (morsicatio linguarum) também podem ser afetadas (AMADORI F,et al. 2018).

A mucosa mordiscada é uma circunstância ocasionada pela mastigação habitual da mucosa jugal, no qual apresentam-se clinicamente como lesões brancas e fragmentadas, podendo manifestar zonas eritematosas com ulceração traumática focal ou erosão, com superfície dilacerada e irregular. Uma mucosa afetada exibe condição retalhada bilateral combinada com lesões de lábios e língua, embora possa se apresentar unilateralmente, conforme os hábitos parafuncionais que o paciente apresente. Essas lesões são por vezes distintas o suficiente para um diagnóstico baseado apenas em características clínicas, sendo então suficientes para o diagnóstico, dessa forma raramente há necessidade de exames complementares (SANTOSWB, et al., 2018).

Encontram-se maior predominância de mucosa mordiscada em pessoas estressadas ou psicologicamente debilitadas, apresentando maior prevalência na segunda e terceira década de vida e em pacientes jovens, no entanto não apresenta predileção por gênero (VAN DER WAL JE, 2018).

Um correto diagnóstico pode ajudar a evitar as lesões, inclusive a importância do diagnóstico diferencial com outras doenças dermatológicas que envolvem a cavidade oral como: líquen plano, mucosa benigna, penfigóide, linha alba ou nevo esponjoso oral (NEVILLE B, et al., 2016). É importante lançar mão de medidas terapêuticas adequadas para o tratamento e o treinamento do paciente para a reversão de hábitos, 0 psiquiatra e/ou psicólogo deve trabalhar em controle com o cirurgião-dentista gerenciando na intervenção comportamental projetada para reduzir as manifestações de distúrbios baseados em hábitos (GAMA E, et al., 2013).

As manifestações orais da mucosa mordiscada podem se apresentar em consequência da ansiedade, que se caracterizam frente a situações ameaçadoras, sentimentos de incerteza, impotência, medo ou dúvida (CASTILLO AR, et al., 2000). Consequentemente, a mucosa mordiscada pode surgir como maneira de "escape" para e exteriorizar emoções e estímulos reprimidos (GAMA E, et al., 2013).

Ansiedade é uma intuição de vazio, sensação de temor e receio, descrita por nervosismo, preocupação ou incômodo originário de antecipação de risco de algo oculto ou suspeito. Os aspectos patológicos reconhecidos de ansiedade e medo são demasiados, incompatíveis em associação aos impulsos, ou qualitativamente distintos do que se analisa como padrão em uma faixa etária, interferindo no bem-estar, estado emocional ou na atividade diária do sujeito (CASTILLO AR, et al., 2000).

Os fatores relevantes que se relacionam ao propósito da automutilação são cessar ou satisfazer as tensões emocionais (angústia, tristeza, alegria, medo, culpa e prazer) (VIEIRA MG, et al., 2016). A apreciação pela dor física do indivíduo permite desviar sua tensão psíquica, resultando numa substituição de tensão biológica 
resulte no retorno impulsivo para a autodestruição corporal (ALMEIDARS, et al., 2018) que resulta numa sensação de bem-estar e alívio, podendo perdurar por horas ou dias, com iminente regresso do ato autodestrutivo (GIUSTI JS, 2013).

A prevalência de automutilação na maioria dos casos, ocorre em mulheres, adolescentes, adultos, prisioneiros e pacientes com distúrbios psiquiátricos, visivelmente naqueles indivíduos que apresentam personalidade de bordeline, distúrbios alimentares, transtorno de estresse pós-traumático, depressão, transtorno de ansiedade e histórico de pessoas que foram abusadas ou tiveram algum trauma (ARCOVERDE RL e SOARES LSLC, 2012)

Deste modo, a ansiedade e a depressão podem estar associadas à automutilação, visto que, além de expulsar emoções e sentimentos negativos também podem afetar o comportamento do indivíduo em atividades que envolvem a participação das funções executivas. Portanto, a depressão está relacionada a alterações de desatenção como também pouca flexibilidade cognitiva, ao mesmo tempo em que a ansiedade resulta em menores níveis de tolerância a frustração e maior impulsividade. (ARCOVERDE RL e SOARES LSLC, 2012).

O objetivo do presente estudo é relatar um caso de um paciente adolescente, apresentando a mucosa mordiscada relacionada à possível automutilação, discutir as características clínicas e a conduta adequada para esse tipo de lesão.

\section{DETALHAMENTE DO CASO}

Paciente do gênero masculino, 15 anos e feoderma, compareceu ao serviço de Odontologia do Centro Universitário Cesmac, Maceió-AL. Durante a anamnese relatou como queixa principal "Médica me encaminhou para fazer uma biópsia". Na história da doença atual não houve relatos de comprometimentos sistêmicos, porém o paciente relatou mordiscar a mucosa jugal bilateralmente, lábio inferior e língua de forma voluntária (Figura $1 \mathrm{~A}$ e B).

Figura 1 - A: Aspecto do lábio inferior, B: Marcas em ventre de língua e mucosa jugal decorrente da mordida do paciente.

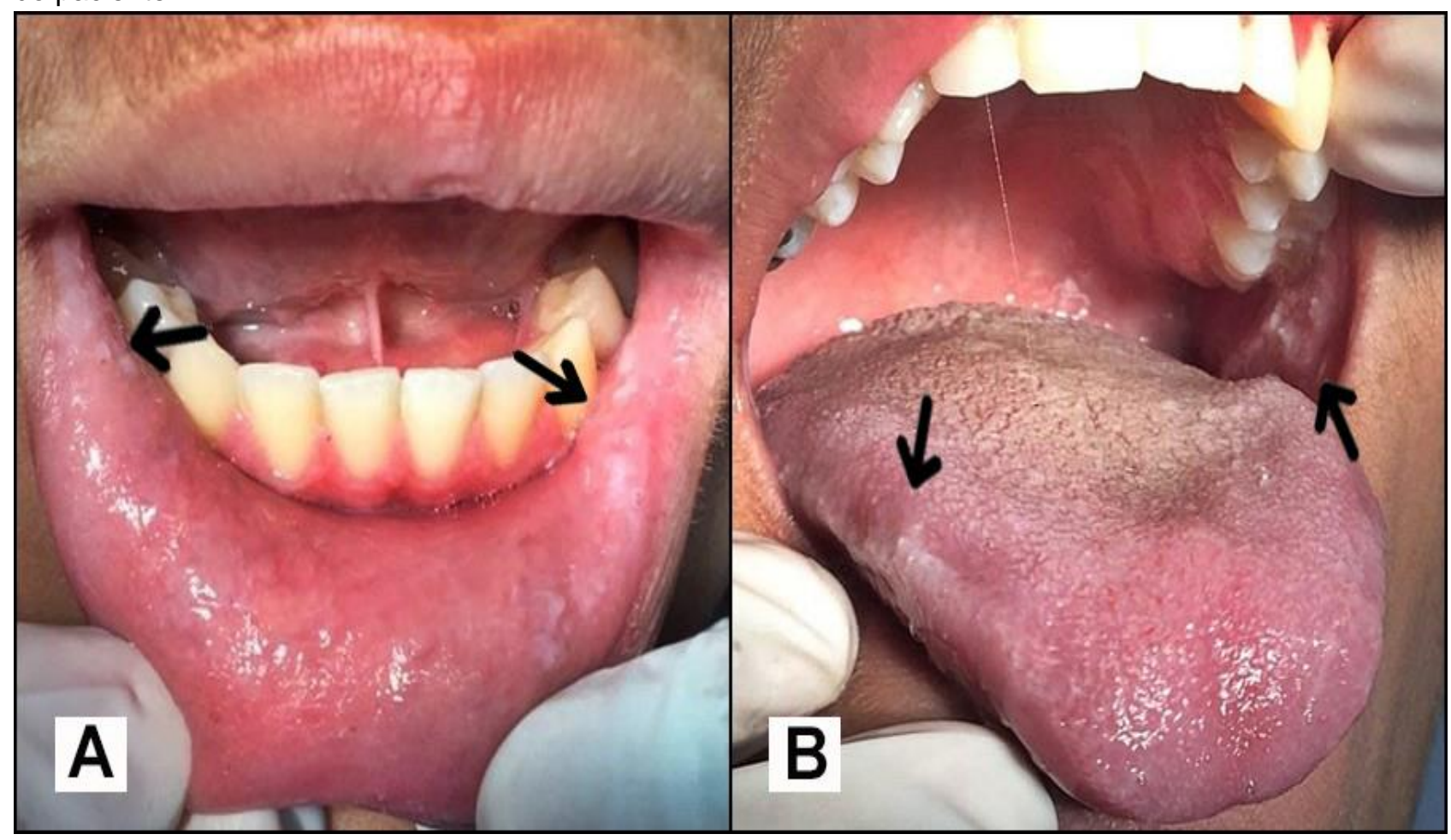

Fonte: Santos WB, et al., 2019. 
Durante o exame físico, observou-se a pressão arterial de 120x80 milímetros de mercúrio (mmHg), frequência respiratória de 18 incursos e frequência cardíaca de 80 batimentos por minuto. Apesar dos parâmetros de normalidade dos sinais vitais, o paciente demonstrava comportamento típico de um indivíduo ansioso, como tensão e preocupação excessiva durante o atendimento apresentando o hábito de mordiscar a mucosa jugal e lábio inferior.

Ao exame extrabucal não houve presença de linfonodos palpáveis ou assimetria facial e não apresentava sinais e sintomas da disfunção temporomandibular. No exame clínico intraoral, observou-se a língua saburrosa, decorrente da má higienização da língua e mucosa mordiscada, apresentando-se como uma placa branco-acinzentada não removível a raspagem e assintomática, localizada em porção média da mucosa jugal bilateralmente e no lábio inferior. Além da mucosa mordiscada, o paciente apresentava a linha alba bem evidente, compatível com o quadro do hábito parafuncional presente (Figura 2 A e B). Não foi necessária a realização de exames complementares como a biópsia, devido às características clínicas da lesão e fatores relacionados à etiologia.

Figura 2 - A e B: Aspecto bilateral da Mucosa jugal mordiscada e Linha Alba.

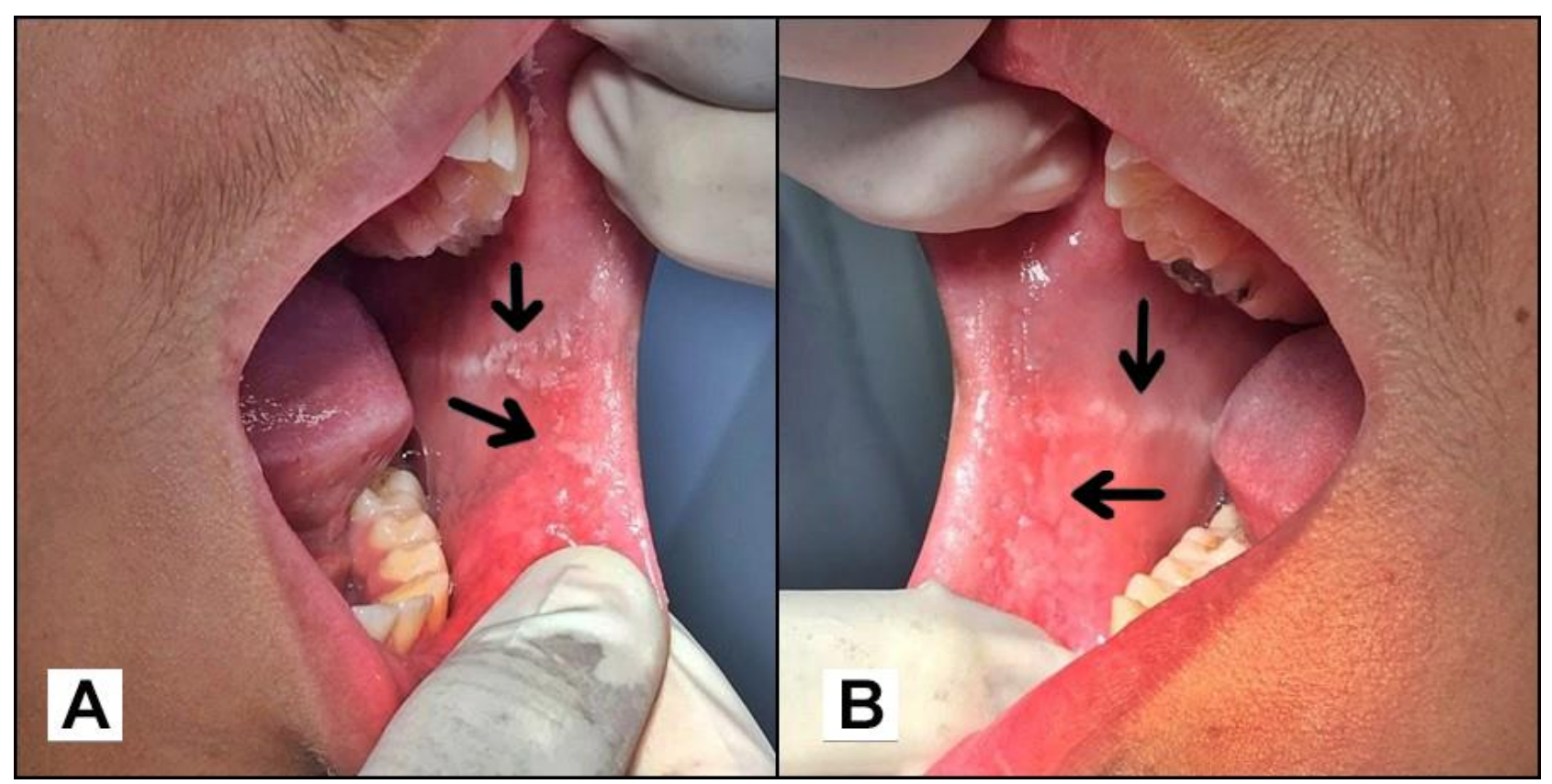

Fonte: Santos WB, et al., 2019.

A conduta proposta foi à realização de instrução e motivação de higiene oral, prescrição de pomada para uso tópico à base de triancinolona acetonida bem como informações sobre a etiologia da doença, a fim de conscientizar o paciente a cessar os hábitos parafuncionais para a regressão da lesão. $O$ paciente foi encaminhado à clínica de psicologia para avaliação e controle do possível transtorno de ansiedade.

\section{DISCUSSÃO}

A mucosa mordiscada é uma condição causada por irritação física crônica, como mordida contínua e frequente da língua, lábios e mucosa, acometendo geralmente pacientes ansiosos e tensos, que também podem apresentar hábitos parafuncionais associados disfunções temporomandibulares ou distúrbios psicológicos (KANG HS, et al., 2012). Neste caso, o paciente apresentava comportamento ansioso e relatava que habitualmente mordiscava a mucosa jugal, língua e lábios com os dentes.

Esse frequente hábito de mordiscar os tecidos bucais produz lesões que são mais comumente encontradas na mucosa jugal; entretanto, a mucosa labial (morsicatio labiorum) e a borda lateral da língua 
(morsicatio linguarum) também são sítios que podem estar envolvidos. São clinicamente caracterizadas como as áreas hiperqueratóticas espessas e fragmentadas de forma irregular. No entanto, focos de erosão, eritema ou ainda áreas de ulceração traumática, podem ocasionalmente encontrados. (MIN KW, et al., 2009).

Os locais acometidos do presente caso estão em concordância com a literatura, no qual se observou lesões características em mucosa jugalbilateral, lábio inferior e borda lateral esquerda da língua. Em pacientes mais jovens, este hábito estava atribuído às reações psicológicas de ansiedade ou automutilação. Em contrapartida, pacientes com a idade acima dos 50 anos, essas lesões estavam associadas ao uso de próteses totais ou parciais (SANTIAGO FC, 2015).

A apreciação pela dor física do indivíduo permite desviar sua tensão psíquica, resultando numa substituição de tensão biológica pelo retorno impulsivo para a autodestruição corporal, estabelecendo uma sensação de bem-estar e alívio, podendo perdurar por horas ou dias, com iminente regresso do ato autodestrutivo. Este hábito é normalmente inconsciente, no entanto poderão ser inúmeros os motivos na origem deste processo de automutilação, sendo mencionada por vários autores a possível influência da ansiedade e estresse emocional. (GIUSTI JS, 2013; LIMA LCMP, 2010). Concordando com a literatura, o paciente demonstrava-se ansioso, relacionando uma possível influência do aspecto emocional relacionada ao desenvolvimento da mucosa mordiscada.

Podem ser encontrados diferentes aspectos clínicos, sendo mais frequente o surgimento de placas brancas e irregulares associadas a uma descamação epitelial. Essas características devem ser observadas com cautela, no qual algumas delas podem se distinguir de lesões leucoplásicas, como por exemplo a superfície descamativa, a rugosidade da lesão e afetando mais comumente a mucosa jugal bilateral, onde não há um limite nítido entre a área de mucosa normal e a afetada, podendo ocasionar danos isolados ou de forma conjunta com a língua e lábios (FRIKHA F, et al., 2018). Essas lesões orais ainda são pouco conhecidas, logo ressalta-se a importância do diagnóstico diferencial, principalmente com as lesões brancas, evitando possíveis erros de conduta.

As características clínicas da mucosa mordiscada são suficientes para o diagnóstico na maioria das vezes, e a biópsia raramente é realizada. Não há nenhuma complicação nem tampouco necessidade de tratamento, obtendo-se um padrão de normalidade do local afetado logo após a suspensão do hábito. Para promover a resolução rápida das lesões, bem como apresentar resultados estéticos aceitáveis sem interferir na fala, podem ser confeccionados placas com proteção bilateral acrílica, por restringir o acesso à mucosa jugal e labial, para aqueles pacientes que necessitam confirmar da causa. Há autores que também sugerem a psicoterapia como o tratamento de escolha, porém não há relatos de benefício com essa terapia (DE MOURA $M$, et al., 2016). Dessa forma, o diagnóstico do presente caso foi somente clínico, não havendo necessidade de biópsia. A avaliação de um psicólogo para determinar se o paciente possui ansiedade é um instrumento válido, assim, tornando-se necessário para que o tratamento possa ser conduzido de forma correta e interdisciplinar.

As lesões de mucosa mordiscada apresentaram índice de 2,5\% em populações caucasianas. Intervenções geralmente são desnecessárias, é recomendado somente cessar o hábito parafuncional. No entanto, quando necessário, podemos lançar mão da confecção da placa com material acrílico, para ser utilizada na superfície oclusal dos dentes evitando o contato involuntário. Porém, o paciente estar ciente da condição compulsiva que apresenta é o método mais eficaz para conseguir obter a remoção do hábito e consequentemente a regressão da lesão (KORAY M e TOSUN T, 2019). No presente caso, o paciente foi orientado quanto a etiologia das lesões, foi prescrito a aplicação de corticosteróide tópicoe então solicitado o acompanhamento psicológico pelo possível transtorno de ansiedade.

$\mathrm{Na}$ atualidade considera-se que a inflamação rotineira e persistente em determinada região, pode suscitar ao surgimento do câncer. Devido ao fato que através de injúrias físicas ou químicas, patologias autoimunes, infecções e radiações, propicie ou induza a carcinogênese, por danificarem o DNA, estimulando assim, a multiplicação celular e dispersão de citocinas e fatores de crescimento (PIEMONTE ED, et al., 2018). Neste relato de caso, a irritação mecânica foi diagnosticada e o paciente foi orientado a cessar os presentes hábitos parafuncionais. 
No estudo de Piemonte ED, et al. (2018), a prevalência das irritações mecânicas crônicas foi de $36 \%$ em grupo controle (composta por pessoas que procuram atendimento odontológico e que oferecem condições para a ocorrência das lesões), caracterizada como uma circunstância oral comum. Porém, no grupo Estudo (casos de carcinoma de células escamosas), seu acometimento foi de $72 \%$, identificando ser um fator do risco predominante em grau superior ao consumo de tabaco e álcool. Apesar do estado de etilistas e/ou tabagistas, as irritações mecânicas crônicas se detectaram relevantes em relação as estatísticas do câncer oral, em multiplas anáslises. Tais informações podem indicar que a irritação mecânica crônica pode exercer não apenas um fator de risco de câncer bucal, tal qual pode ser apontado como um distúrbio potencialmente maligno da mucosa oral.

De acordo com os resultados obtidos e metodologia empregada, conclui-se que pacientes com quadros de alterações psicológicas podem desenvolver alterações na cavidade bucal em virtude dos transtornos emocionais, como o emprego de hábitos parafuncionais, que geralmente levam aos portadores agerar lesões como a mucosa mordiscada. É de grande importância que o cirurgião-dentista seja capaz de reconhecer e tratar os sintomas de forma multiprofissional. A necessidade de acompanhamento psicológico, adjunto as medidas propostas para o tratamento, podem viabilizar a condição do paciente a diminuir ou cessar os hábitos autolesivos.

\section{REFERÊNCIAS}

1. ALMEIDA RS, et al. A prática da automutilação na adolescência: o olhar da psicologia escolar/educacional. Ciências Humanas e Sociais, 2018; 4(3): 147-160.

2. AMADORI F, et al. Oral mucosal lesions in teenagers: a cross-sectional study. Italian journal of pediatrics, 2017; 43(1):50.

3. ARCOVERDE RL, SOARES LSLC. Funções neuropsicológicas associadas a condutas autolesivas: revisão integrativa de literatura. Psicologia: Reflexão e Crítica, 2011; 25(2): 293-300.

4. CASTILLO AR, et al. Transtorno de Ansiedade. Jornal Brasileiro de Psiquiatria, 2018;22: 20-23.

5. DE MOURA M, et al.MorsicatioBuccarum: relato de caso clínico. Revista de Iniciação Científica da Universidade Vale do Rio Verde, 2016; 6(2).

6. FRIKHA F, et al. Morsicatio buccarum et labiorum: diagnostic simple parfois déroutant. La Revue de Médecine Interne,2018; 39:182.

7. GAMA E, et al. Bruxismo: revisão de literatura. Revista Científica Multidisciplinar das Faculdades de São José, 2013 ; 1(1): 16-22.

8. GIUSTI, JS. Automutilação: características e comparação com pacientes com transtorno obsessivo-compulsivo. Dissertação (Doutorado em Medicina) - Faculdade de Medicina. Universidade de São Paulo, São Paulo, $2013 ; 184$ p.

9. KANG HS, et al. Three Cases ofMorsicatioLabiorum. AnnalsofDermatology, 2012; 24(4): 455-458.

10. KORAY M, TOSUN, T. Oral Mucosal Trauma and Injuries. Trauma in Dentistry. IntechOpen, 2019.

11. LIMA, LCMP. MorsicatioBuccarum e possíveis factores etiológicos. Dissertação (Mestrado em Medicina Dentária) Instituto de Ciências da Saúde. UniversidadeCatólica Portuguesa, Viseu, 2010; 58 p.

12. MIN KW, PARK CK. Three Cases Report and a Review of the Literature. The Korean Journal of Pathology, 2009; 43(2): 174-176.

13. NEVILLE B, et al. Patologia oral e maxilofacial. 4 ed, Elsevier Brasil, 2016; 928 p.

14. PIEMONTE ED, et al. Oral cancer associated with chronic mechanical irritation of the oral mucosa. Medicina Oral Patologia Oral y Cirurgia Bucal, 2018; 23(2): 151-160.

15. SANTIAGO, FC. Hábitos orais parafuncionais: uma revisão da literatura. Dissertação (Mestrado em Odontologia) Faculdade de Odontologia. Universidade Federal do Rio de Janeiro, Rio de Janeiro, 2015; 50 p.

16. SANTOS WB, et al. Bruxismo e mucosa mordiscada relacionada à possível ansiedade: relato de caso. Revista da Academia Brasileira de Odontologia, 2018; 8(2): 42-46.

17. VAN DER WAL JE. Morsicatio Mucosae Oris. Encyclopedia of Pathology. Springer, Cham, 2018; p. 1-2.

18. VIEIRA MG, et al. Automutilação: intensidade dolorosa, fatores desencadeantes e gratificantes. Revista Dor, 2016; 17(4): 257-260. 